\title{
Erratum to: Conformal and continuous deposition of bifunctional cobalt phosphide layers on p-silicon nanowire arrays for improved solar hydrogen evolution
}

Sitaramanjaneya Mouli Thalluri ${ }^{1}$, Jerome Borme ${ }^{1}$, Kang $\mathrm{Yu}^{1,2}$, Junyuan $\mathrm{Xu}^{1}$, Isilda Amorim ${ }^{1}$, Joao Gaspar ${ }^{1}$, Liang Qiao ${ }^{3}$, Paulo Ferreira ${ }^{1,2,4}$, Pedro Alpuim ${ }^{1,5}$, and Lifeng Liu ${ }^{1}(\bowtie)$

${ }^{1}$ International Iberian Nanotechnology Laboratory (INL), Av. Mestre. Jose Veiga, Braga 4715-330, Portugal

${ }^{2}$ Materials Science and Engineering Program, University of Texas at Austin, Austin TX78712, USA

${ }^{3}$ Department of Chemistry, State Key Laboratory of Molecular Engineering of Polymers and Institute of Biomedical Sciences, Fudan University, Shanghai 200433, China

${ }^{4}$ Mechanical Engineering Department and IDMEC, Instituto Superior Técnico, University of Lisbon, Av. Rovisco Pais, Lisboa 1049-001, Portugal

${ }^{5}$ Center of Physics, University of Minho, Braga 4710-057, Portugal

(C) Tsinghua University Press and Springer-Verlag GmbH Germany, part of Springer Nature 2020

\section{Erratum to}

Nano Research 2018, 11(9): 4823-4835

https://doi.org/10.1007/s12274-018-2070-4

The name of the first author was unfortunately misspelled,

Instead of

Sitaramanjaneva Mouli Thalluri
It should read

Sitaramanjaneya Mouli Thalluri

The online version of the original article can be found at

https://doi.org/10.1007/s12274-018-2070-4 